\title{
LINEARIZATIONS OF ORDINARY DIFFERENTIAL EQUATIONS BY AREA PRESERVING MAPS
}

\author{
TETSUYA OZAWA ${ }^{1}$ AND HAJIME SATO ${ }^{2}$
}

\begin{abstract}
We clarify the class of second and third order ordinary differential equations which can be tranformed to the simplest equations $Y^{\prime \prime}=0$ and $Y^{\prime \prime \prime}=0$. The coordinate changes employed to transform the equations are respectively area preserving maps for second order equations and contact form preserving maps for third order equations. A geometric explanation of the results is also given by using connections and associated covariant differentials both on tangent and cotangent spaces.
\end{abstract}

\section{$\S 1$. Introduction}

In this paper, we consider second and third order ordinary differential equations, and investigate the conditions under which those equations are transformed to the simplest equations $Y^{\prime \prime}=0$ and $Y^{\prime \prime \prime}=0$, where the coordinate changes used to transform equations are area preserving maps for second order equations and contact form preserving maps for third order equations.

The equivalence problem of ordinary differential equations are classified into several categories, depending on the diffeomorphisms used to transform the equations. An interesting version of the problem employs point transformations $(x, y) \mapsto(X(x, y), Y(x, y))$, and the study of second order equations in this category was originated by Tresse in [1]. In [2] and [3], Cartan gave a geometric interpretation of Tresse's results in terms of a projective connection. For a resent works, see also Kamran-Lamb-Shadwick [5], who discussed the equivalence problem under fiber preserving diffeomorphims $(x, y) \mapsto(X(x), Y(x, y))$.

Cartan also studied third order equations under the equivalence by point transformations $(x, y) \mapsto(X(x, y), Y(x, y))$ in [4]. On the other hand,

\footnotetext{
Received April 27, 1998.

${ }^{1}$ This research was partially supported by Grant-inAid for Scientific Research (No. 09640136), Miniatry of Education Science and Culture, Japan.

${ }^{2}$ This research was partially supported by Grant-inAid for Scientific Research (No. 08454016), Miniatry of Education Science and Culture, Japan.
} 
Chern treated the problem with contact transformations $\left(x, y, y^{\prime}\right) \mapsto$ $\left(X\left(x, y, y^{\prime}\right), Y\left(x, y, y^{\prime}\right), Y^{\prime}\left(x, y, y^{\prime}\right)\right)$, in [6]. In [7] and [8], Sato and Yoshikawa completed the Cherns works on this category.

Our main theorems are the following:

THEOREM 1.1. An ordinary differential equation $y^{\prime \prime}+f\left(x, y, y^{\prime}\right)=0$ can be transformed to the equation $Y^{\prime \prime}=0$ by an area preserving map $(x, y) \mapsto(X, Y)$, if and only if $f(x, y, z)$ is a polynomial of degree 3 in the variable $z$;

$$
f=P+3 Q z+3 R z^{2}+S z^{3}
$$

and $P, Q, R$ and $S$ satisfy the equations

$$
\left\{\begin{array}{l}
P_{y}-Q_{x}=-2\left(P R-Q^{2}\right) \\
Q_{y}-R_{x}=-P S+Q R \\
R_{y}-S_{x}=-2\left(Q S-R^{2}\right) .
\end{array}\right.
$$

TheOREM 1.2. An ordinary differential equation $y^{\prime \prime \prime}+f\left(x, y, y^{\prime}, y^{\prime \prime}\right)=$ 0 can be transformed to the equation $Y^{\prime \prime \prime}=0$ by a contact form preserving map $(x, y, z) \mapsto(X, Y, Z)$, if and only if $f(x, y, z, w)$ is a polynomial of degree 3 in the variable $w$;

$$
f=P+3 Q w+3 R w^{2}+S w^{3}
$$

and the functions $P, Q, R$ and $S$ depend only on $x$ and $z$, and satisfy the system of equations

$$
\left\{\begin{array}{l}
P_{z}-Q_{x}=-2\left(P R-Q^{2}\right) \\
Q_{z}-R_{x}=-P S+Q R \\
R_{z}-S_{x}=-2\left(Q S-R^{2}\right) .
\end{array}\right.
$$

By Lemma 3.2 in which we prove that contact form preserving maps are lifts of area preserving maps, we reduce the proofs of Theorem 1.1 and 1.2 to the same integrability condition, and therefore in both theorems we have the same system of partial differential equations (1) and (2)

In Section 4, we interpret the integrability conditions in Theorem 1.1 and 1.2 as a flatness of a certain connection form, and show how to construct the required coordinate change using the dual covariant differential associated with the connection form. 


\section{$\S 2$. Second order ordinary differential equation}

In this section, we consider second order ordinary differential equations

$$
y^{\prime \prime}+f\left(x, y, y^{\prime}\right)=0,
$$

and their transformations by area preserving maps.

\subsection{Transformations of equations}

Being the $(x, y, z)$-space $\mathbf{R}^{3}$ regarded as a contact manifold whose contact form is equal to $d y-z d x$, for a diffeomorphism $\varphi: U \rightarrow V$ between open sets $U$ and $V$ in $\mathbf{R}^{2}$, there exists a unique contact tranformation $\Phi: \mathbf{R}^{3} \rightarrow \mathbf{R}^{3}$ which is a lift of $\varphi$ with respect to the projection $(x, y, z) \mapsto(x, y)$. If we write $\varphi(x, y)=(X(x, y), Y(x, y))$, then $\Phi$ is given by

$$
\Phi(x, y, z)=\left(X(x, y), Y(x, y), Z=\frac{Y_{x}+Y_{y} z}{X_{x}+X_{y} z}\right) .
$$

Let $\tilde{c}:(a, b) \rightarrow \mathbf{R}^{3}$ be the Legendrean lift of the graph of a function $y=y(x)$. Then along the image curve $\Phi \circ \tilde{c}$, it holds that

$$
\begin{aligned}
\frac{d Z}{d X} & =\frac{d Z}{d x} / \frac{d X}{d x} \\
& =\frac{\Delta}{\left(X_{x}+X_{y} y^{\prime}\right)^{3}}\left[P+3 Q y^{\prime}+3 R\left(y^{\prime}\right)^{2}+S\left(y^{\prime}\right)^{3}+y^{\prime \prime}\right],
\end{aligned}
$$

where $P, Q, R$ and $S$ are functions of $x$ and $y$ given by

$$
\left\{\begin{aligned}
\Delta P & =Y_{x x} X_{x}-X_{x x} Y_{x} \\
3 \Delta Q & =Y_{x x} X_{y}+2 Y_{x y} X_{x}-X_{x x} Y_{y}-2 X_{x y} Y_{x} \\
3 \Delta R & =2 Y_{x y} X_{y}+Y_{y y} X_{x}-2 X_{x y} Y_{y}-X_{y y} Y_{x} \\
\Delta S & =Y_{y y} X_{y}-X_{y y} Y_{y},
\end{aligned}\right.
$$

and $\Delta=Y_{y} X_{x}-Y_{x} X_{y}$. This shows the following

LEMma 2.1. In order that the equation

$$
y^{\prime \prime}+f\left(x, y, y^{\prime}\right)=0,
$$

can be transformed to the equation

$$
Y^{\prime \prime}=0
$$

the function $f(x, y, z)$ has to be a polynomial of order 3 in the variable $z$;

$$
f(x, y, z)=P+3 Q z+3 R z^{2}+S z^{3} .
$$




\subsection{The integrability condition}

Now, we consider the following equivalence problem: what is the necessary and sufficient condition on $P, Q, R$ and $S$, under which the equations (4) and (5) can be transformed to each other through the natural lifts of area preserving maps.

Let $\varphi(x, y)=(X, Y)$ be an area preserving map. Differentiating $\Delta=$ $X_{x} Y_{y}-X_{y} Y_{x} \equiv 1$ by $x$ and $y$, we get

$$
\begin{aligned}
& 0=X_{x x} Y_{y}+X_{x} Y_{y x}-X_{x y} Y_{x}-X_{y} Y_{x x} \\
& 0=X_{x y} Y_{y}+X_{x} Y_{y y}-X_{y y} Y_{x}-X_{y} Y_{x y} .
\end{aligned}
$$

Combining these two identities with four equalities (3), we obtain the following system of equations:

$$
\left(\begin{array}{c}
P \\
3 Q \\
3 R \\
S \\
0 \\
0
\end{array}\right)=\left(\begin{array}{cccccc}
X_{x} & 0 & 0 & -Y_{x} & 0 & 0 \\
X_{y} & 2 X_{x} & 0 & -Y_{y} & -2 Y_{x} & 0 \\
0 & 2 X_{y} & X_{x} & 0 & -2 Y_{y} & -Y_{x} \\
0 & 0 & X_{y} & 0 & 0 & -Y_{y} \\
-X_{y} & X_{x} & 0 & Y_{y} & -Y_{x} & 0 \\
0 & -X_{y} & X_{x} & 0 & Y_{y} & -Y_{x}
\end{array}\right)\left(\begin{array}{c}
Y_{x x} \\
Y_{x y} \\
Y_{y y} \\
X_{x x} \\
X_{x y} \\
X_{y y}
\end{array}\right)
$$

and consequently the relation

$$
\left(\begin{array}{c}
Y_{x x} \\
Y_{x y} \\
Y_{y y} \\
X_{x x} \\
X_{x y} \\
X_{y y}
\end{array}\right)=\left(\begin{array}{cccccc}
Y_{y} & -Y_{x} & 0 & 0 & 0 & 0 \\
0 & 0 & 0 & 0 & Y_{y} & -Y_{x} \\
0 & 0 & Y_{y} & -Y_{x} & 0 & 0 \\
X_{y} & -X_{x} & 0 & 0 & 0 & 0 \\
0 & 0 & 0 & 0 & X_{y} & -X_{x} \\
0 & 0 & X_{y} & -X_{x} & 0 & 0
\end{array}\right)\left(\begin{array}{c}
P \\
Q \\
R \\
S \\
Q \\
R
\end{array}\right) .
$$

These are second order partial differential equations on $X$ and $Y$. We remark that $X$ and $Y$ satisfy the same equations, namely both $X$ and $Y$ satisfy

$$
\left\{\begin{array}{l}
X_{x x}=X_{y} P-X_{x} Q \\
X_{x y}=X_{y} Q-X_{x} R \\
X_{y y}=X_{y} R-X_{x} S
\end{array}\right.
$$

The compatibility condition among these equations

$$
\frac{\partial}{\partial y}\left(X_{x x}\right)=\frac{\partial}{\partial x}\left(X_{x y}\right), \quad \frac{\partial}{\partial y}\left(X_{x y}\right)=\frac{\partial}{\partial x}\left(X_{y y}\right)
$$


will impose the following relations

$$
\begin{aligned}
0= & \frac{\partial}{\partial y}\left(X_{x x}\right)-\frac{\partial}{\partial x}\left(X_{x y}\right) \\
= & \frac{\partial}{\partial y}\left(X_{y} P-X_{x} Q\right)-\frac{\partial}{\partial x}\left(X_{y} Q-X_{x} R\right) \\
= & X_{y y} P-X_{x y} Q+X_{y} P_{y}-X_{x} Q_{y} \\
& \quad-X_{x y} Q+X_{x x} R-X_{y} Q_{x}+X_{x} R_{x} \\
= & \left(X_{y} R-X_{x} S\right) P-\left(X_{y} Q-X_{x} R\right) Q+X_{y} P_{y}-X_{x} Q_{y} \\
& \quad-\left(X_{y} Q-X_{x} R\right) Q+\left(X_{y} P-X_{x} Q\right) R-X_{y} Q_{x}+X_{x} R_{x} \\
= & -X_{x}\left(Q_{y}-R_{x}+P S-Q R\right)+X_{y}\left(P_{y}-Q_{x}+2 P R-2 Q^{2}\right)
\end{aligned}
$$

(9) $\quad 0=\frac{\partial}{\partial y}\left(X_{x y}\right)-\frac{\partial}{\partial x}\left(X_{y y}\right)$

$$
\begin{aligned}
= & \frac{\partial}{\partial y}\left(X_{y} Q-X_{x} R\right)-\frac{\partial}{\partial x}\left(X_{y} R-X_{x} S\right) \\
= & X_{y y} Q-X_{x y} R+X_{y} Q_{y}-X_{x} R_{y} \\
& \quad-X_{x y} R+X_{x x} S-X_{y} R_{x}+X_{x} S_{x} \\
= & \left(X_{y} R-X_{x} S\right) Q-\left(X_{y} Q-X_{x} R\right) R+X_{y} Q_{y}-X_{x} R_{y} \\
& \quad-\left(X_{y} Q-X_{x} R\right) R+\left(X_{y} P-X_{x} Q\right) S-X_{y} R_{x}+X_{x} S_{x} \\
= & -X_{x}\left(R_{y}-S_{x}+2 Q S-2 R^{2}\right)+X_{y}\left(Q_{y}-R_{x}+P S-Q R\right) .
\end{aligned}
$$

Lemma 2.2. In order that there exists a coordinate change $(X, Y)$ which preserves the area elements and satisfies the equation (7), it is necessary and sufficient that $P, Q, R$ and $S$ satisfy the system of equations

$$
\left\{\begin{array}{l}
P_{y}-Q_{x}=-2\left(P R-Q^{2}\right) \\
Q_{y}-R_{x}=-P S+Q R \\
R_{y}-S_{x}=-2\left(Q S-R^{2}\right) .
\end{array}\right.
$$

Proof. If the map $(X, Y)$ is a coordinate change, then the derivatives $\left(X_{x}, X_{y}\right)$ and $\left(Y_{x}, Y_{y}\right)$ are linearly independent. Thus if the coordinate functions $X$ and $Y$ satisfy (7), then the coeffecients of $X_{x}$ and $X_{y}$ in the expressions (8) and (9) are equal to zero.

On the other hand, if the equations (10) are satisfied, then the compatibility conditions of the equations (7) are satisfied. Then we see that 
there exists an area preserving map $(X, Y)$ which satisfies (7). A geometric explanation of the map $(X, Y)$ will be given in Section 4 .

THEOREM 2.1. An ordinary differential equation $y^{\prime \prime}+f\left(x, y, y^{\prime}\right)=0$ can be transformed to the equation $Y^{\prime \prime}=0$ by an area preserving map $(x, y) \mapsto(X, Y)$, if and only if $f(x, y, z)$ is a polynomial of degree 3 in the variable $z$;

$$
f=P+3 Q z+3 R z^{2}+S z^{3}
$$

and $P, Q, R$ and $S$ satisfy the equations (10).

\section{$\S 3$. Third order ordinary differential equation}

In this section, we consider third order ordinary differential equations

$$
y^{\prime \prime \prime}+f\left(x, y, y^{\prime}, y^{\prime \prime}\right)=0
$$

and their transformations by contact form preserving maps.

\subsection{Transformations of third order ordinary differential equations}

Let the $(x, y, z, w)$-space be regarded as the third order jet space $\left(x, y, y^{\prime}, y^{\prime \prime}\right)$. For a contact diffeomorphism $\varphi:(x, y, z) \mapsto(X, Y, Z)$, let $\Phi:(x, y, z, w) \mapsto(X, Y, Z, W)$ denote the canonical lift of $\varphi$;

$$
\Phi(x, y, z, w)=\left(X, Y, Z, W=\frac{Z_{x}+Z_{y} z+Z_{z} w}{X_{x}+X_{y} z+X_{z} w}\right) .
$$

Along the image curve $\Phi \circ \tilde{c}$ where $\tilde{c}(x)=\left(x, y(x), y^{\prime}(x), y^{\prime \prime}(x)\right)$, we calculate $\frac{d W}{d X}$ as follows: using the notation

$$
\llbracket A, B \rrbracket=Z_{A} X_{B}-Z_{B} X_{A}
$$

for $A, B=x, y, z, x x, x y, \cdots, z z$, and

$$
\begin{aligned}
& \eta(\alpha \beta, \gamma)=\text { the number of } y \text { 's in }\{\alpha, \beta, \gamma\} \\
& \zeta(\alpha \beta, \gamma)=\text { the number of } z \text { 's in }\{\alpha, \beta, \gamma\}
\end{aligned}
$$

$\frac{d W}{d X}$ is equal to

$$
\frac{d W}{d X}=\frac{d W}{d x} / \frac{d X}{d x}
$$




$$
\begin{aligned}
& =\frac{d}{d x}\left(\frac{Z_{x}+Z_{y} z+Z_{z} w}{X_{x}+X_{y} z+X_{z} w}\right) /\left(X_{x}+X_{y} z+X_{z} w\right) \\
& =\frac{1}{\left(X_{x}+X_{y} z+X_{z} w\right)^{3}}[* *]
\end{aligned}
$$

where we denote

$$
\begin{aligned}
{[* *]=} & \sum \llbracket \alpha \beta, \gamma \rrbracket z^{\eta(\alpha \beta, \gamma)} w^{\zeta(\alpha \beta, \gamma)} \\
& +\llbracket y, x \rrbracket w+\llbracket y, z \rrbracket w^{2}+(\llbracket z, x \rrbracket+\llbracket z, y \rrbracket z) w^{\prime},
\end{aligned}
$$

and, in the summation $\sum, \alpha \beta$ and $\gamma$ run over the set $\{x x, x y, x z, y y, y z, z z\}$ and $\{x, y, z\}$ respectively.

Thus we obtain

LEMMA 3.1. The equtaion (11) is transformed to the equation

$$
Y^{\prime \prime \prime}=0
$$

by the lift $\Phi$ of a contact transformation $\varphi$, only if the function $f(x, y, z, w)$ is a polynomial of degree three in the variable $w$;

$$
f(x, y, z, w)=P+3 Q w+3 R w^{2}+S w^{3} .
$$

\subsection{Contact form preserving maps}

We investigate maps $\varphi$ which preserve the contact forms, that is, the maps $\varphi(x, y, z)=(X, Y, Z)$ which satisfy

$$
\begin{aligned}
d y-z d x & =d Y-Z d X \\
& =\left(Y_{x}-Z X_{x}\right) d x+\left(Y_{y}-Z X_{y}\right) d y+\left(Y_{z}-Z X_{z}\right) d z
\end{aligned}
$$

For such a map $\varphi$, the coordinate functions $X, Y$ and $Z$ satisfy

$$
\begin{aligned}
& Y_{x}-Z X_{x}=-z \\
& Y_{y}-Z X_{y}=1 \\
& Y_{z}-Z X_{z}=0
\end{aligned}
$$

From the identities $Y_{x y}=Y_{y x}, Y_{y z}=Y_{z y}$ and $Y_{x z}=Y_{z x}$, we get

$$
\begin{aligned}
& Z_{y} X_{x}=Z_{x} X_{y} \\
& Z_{z} X_{y}=Z_{y} X_{z} \\
& Z_{z} X_{x}=Z_{x} X_{z}+1
\end{aligned}
$$


in other words,

$$
\begin{aligned}
0 & =\left|\begin{array}{cc}
X_{x} & X_{y} \\
Z_{x} & Z_{y}
\end{array}\right|=\left|\begin{array}{cc}
X_{y} & X_{z} \\
Z_{y} & Z_{z}
\end{array}\right|, \\
1 & =\left|\begin{array}{cc}
X_{x} & X_{z} \\
Z_{x} & Z_{z}
\end{array}\right| .
\end{aligned}
$$

Since $\left(X_{x}, Z_{x}\right)$ and $\left(X_{z}, Z_{z}\right)$ are linearly independent at each point $(x, y, z)$, this implies

$$
X_{y}=Z_{y}=0
$$

thus $X$ and $Z$ are functions only on the variables $(x, z)$, and the equality $Y_{y} \equiv 1$ implies that $Y$ can be written as

$$
Y(x, y, z)=y+\psi(x, z)
$$

for some function $\psi(x, z)$. Since $\psi$ satisfies

$$
\psi_{x}=-z+Z X_{x}, \quad \psi_{z}=Z X_{z}
$$

$\psi$ can be written as

$$
\psi(x, z)=\int Z d X-z d x .
$$

Therefore we obtain

LEMmA 3.2. If $\varphi:(x, y, z) \mapsto(X, Y, Z)$ preserves the contact form, then $X$ and $Z$ are functions only on the variables $(x, z)$, the transformation $(x, z) \mapsto(X, Z)$ is area preserving, and the function $Y$ is of the form

$$
Y=y+\int Z d X-z d x .
$$

\subsection{Equivalence problem of O.D.E. through contact form preserving maps}

Let $\varphi:(x, y, z) \mapsto(X, Y, Z)$ be a contact form preserving map. We suppose that the equation

$$
0=P+3 Q y^{\prime \prime}+3 R\left(y^{\prime \prime}\right)^{2}+S\left(y^{\prime \prime}\right)^{3}+y^{\prime \prime \prime}
$$

is transformed to the equation

$$
0=Y^{\prime \prime \prime}
$$


by the map $\varphi$. By the formula (12), we see that $P, Q, R$ and $S$ are functions of the derivatives of $X$ and $Z$. Since our functions $X$ and $Z$ depend only on $(x, z)$, we get the following

$$
\begin{aligned}
P & =Z_{x x} X_{x}-X_{x x} Z_{x} \\
3 Q & =2 Z_{x z} X_{x}-2 X_{x z} Z_{x}+Z_{x x} X_{z}-X_{x x} Z_{z} \\
3 R & =2 Z_{x z} X_{z}-2 X_{x z} Z_{z}+Z_{z z} X_{x}-X_{z z} Z_{x} \\
S & =Z_{z z} X_{z}-X_{z z} Z_{z} .
\end{aligned}
$$

This system of equations is completely the same as (3), provided that we use the variables $z$ and $Z$ in place of $y$ and $Y$ in (3). Therefore the necessary and sufficient condition for the existence of the transformation $\varphi$ is the same as that obtained in Theorem 2.1. Thus we proved

THEOREM 3.1. An ordinary differential equation $y^{\prime \prime \prime}+f\left(x, y, y^{\prime}, y^{\prime \prime}\right)=$ 0 can be transformed to a standard equation $Y^{\prime \prime \prime}=0$ by a contact form preserving map $(x, y, z) \mapsto(X, Y, Z)$, if and only if $f(x, y, z, w)$ is a polynomial of degree 3 in the variable $w$;

$$
f=P+3 Q w+3 R w^{2}+S w^{3}
$$

and the functions $P, Q, R$ and $S$ depend only on $x$ and $z$, and satisfy the system of equations

$$
\left\{\begin{array}{l}
P_{z}-Q_{x}=-2\left(P R-Q^{2}\right) \\
Q_{z}-R_{x}=-P S+Q R \\
R_{z}-S_{x}=-2\left(Q S-R^{2}\right) .
\end{array}\right.
$$

\section{$\S 4$. Connections on $\mathbf{R}^{2}$ and the equation of the geodesics}

In this section, we consider the equation of geodesics of a certain type of covariant differentials on the tangent bundle $\mathrm{TR}^{2}$, and reformulate our results in Section 2 using dual covariant differentials on the cotangent bundle $\mathrm{T}^{*} \mathbf{R}^{2}$.

\subsection{Covariant and dual covariant differentials}

Consider $P=\mathbf{R}^{2} \times G L_{2}(\mathbf{R})$ as a principal $G L_{2}(\mathbf{R})$-bundles over $\mathbf{R}^{2}$, on which $G L_{2}(\mathbf{R})$ is acting on the left.

Let $d g g^{-1}$ denote the right invariant Maurer-Cartan form on $G L_{2}(\mathbf{R})$. Then each connection form $\omega \in \Omega^{1}\left(P, g l_{2}(\mathbf{R})\right)$ is decomposed into the vertical and the horizontal components

$$
\omega=d g g^{-1}+\operatorname{Ad}(g) \omega^{h}
$$


where $\omega^{h}$ is a $g l_{2}(\mathbf{R})$-valued 1 -form on $\mathbf{R}^{2}$.

Given a connection form $\omega$, we denote by $\nabla$ the associated covariant differential on the tangent bundle $\mathrm{TR}^{2}$, and by $\nabla^{*}$ the dual covariant differential on the cotangent bundle $T^{*} \mathbf{R}^{2}$.

If we identify a map $\xi=\left(\begin{array}{l}u \\ v\end{array}\right): \mathbf{R}^{2} \rightarrow \mathbf{R}^{2}$ with a section of $\mathrm{TR}^{2}$ by

$$
\xi(x, y)=u(x, y) \frac{\partial}{\partial x}+v(x, y) \frac{\partial}{\partial y},
$$

then $\nabla$ is, by definition, given by

$$
\nabla\left(\begin{array}{l}
u \\
v
\end{array}\right)=d\left(\begin{array}{l}
u \\
v
\end{array}\right)-\omega^{h}\left(\begin{array}{l}
u \\
v
\end{array}\right) .
$$

If we use the Christoffel symbol, $\omega^{h}$ is given by

$$
\omega^{h}=-\left(\begin{array}{ll}
\Gamma_{11}^{1} & \Gamma_{12}^{1} \\
\Gamma_{11}^{2} & \Gamma_{12}^{2}
\end{array}\right) d x-\left(\begin{array}{ll}
\Gamma_{21}^{1} & \Gamma_{22}^{1} \\
\Gamma_{21}^{2} & \Gamma_{22}^{2}
\end{array}\right) d y
$$

The dual covariant differential $\nabla^{*}$ is given by

$$
\nabla^{*}(u, v)=d(u, v)+(u, v) \omega^{h}
$$

where we identified a map $\alpha=(u, v): \mathbf{R}^{2} \rightarrow \mathbf{R}^{2}$ with a section

$$
\alpha(x, y)=u(x, y) d x+v(x, y) d y \in \Gamma\left(\mathrm{T}^{*} \mathbf{R}^{2}\right)=\Omega^{1}\left(\mathbf{R}^{2}\right) .
$$

We remark that the flatness of $\omega$

$$
d \omega^{h}-\omega^{h} \wedge \omega^{h}=0
$$

and that of the corresponding covariant differentials $\nabla$ and $\nabla^{*}$ are all equivalent.

\subsection{Geodesics on $\mathbf{R}^{2}$}

In the following lemma, we will derive the equation of the functions $y=y(x)$ whose graphs are $\nabla$-geodesics on $\mathbf{R}^{2}$.

LEMMA 4.1. The graph of a function $y=y(x)$ is $a \nabla$-geodesic, if and only if $y(x)$ satisfies the ordinary differential equation

$$
y^{\prime \prime}+\Gamma_{11}^{2}+\left(\Gamma_{12}^{2}+\Gamma_{21}^{2}-\Gamma_{11}^{1}\right) y^{\prime}+\left(\Gamma_{22}^{2}-\Gamma_{12}^{1}-\Gamma_{21}^{1}\right)\left(y^{\prime}\right)^{2}-\Gamma_{22}^{1}\left(y^{\prime}\right)^{3}=0
$$


Proof. By definition, a parametrized curve $t \mapsto(x(t), y(t))$ is $\nabla$-geodesic, if it satisfies

$$
\left\{\begin{array}{l}
\frac{d^{2} x}{d t^{2}}=-\left\{+\Gamma_{11}^{1}\left(\frac{d x}{d t}\right)^{2}+\left(\Gamma_{12}^{1}+\Gamma_{21}^{1}\right) \frac{d x}{d t} \frac{d y}{d t}+\Gamma_{22}^{1}\left(\frac{d y}{d t}\right)^{2}\right\} \\
\frac{d^{2} y}{d t^{2}}=-\left\{+\Gamma_{11}^{2}\left(\frac{d x}{d t}\right)^{2}+\left(\Gamma_{12}^{2}+\Gamma_{21}^{2}\right) \frac{d x}{d t} \frac{d y}{d t}+\Gamma_{22}^{2}\left(\frac{d y}{d t}\right)^{2}\right\}
\end{array}\right.
$$

Since $\frac{d y}{d x}=\frac{d y}{d t} / \frac{d x}{d t}$, we have

$$
\begin{aligned}
\frac{d^{2} y}{d x^{2}} & =\frac{d}{d t}\left(\frac{d y}{d t} / \frac{d x}{d t}\right) / \frac{d x}{d t} \\
& =\left(\frac{d^{2} y}{d t^{2}} \frac{d x}{d t}-\frac{d y}{d t} \frac{d^{2} x}{d t^{2}}\right) /\left(\frac{d x}{d t}\right)^{3} .
\end{aligned}
$$

Substituting for $\frac{d^{2} y}{d t^{2}}$ and $\frac{d^{2} x}{d t^{2}}$ the right hand sides of (14), we obtain the required equation.

\subsection{Torsion free flat connection}

LEMMA 4.2. Suppose a connection form $\omega$ is flat, that is, the horizontal component $\omega^{h}$ satisfies

$$
d \omega^{h}-\omega^{h} \wedge \omega^{h}=0
$$

Let $\alpha=(u, v)=u d x+v d y$ be a local $\nabla^{*}$ - parallel section. If $\nabla$ is torsion free, then $\alpha$ is a closed 1-form.

Proof. By definition, $\nabla$ is torsion free, if $\nabla$ satisfies

$$
\nabla_{\xi} \eta-\nabla_{\eta} \xi=[\xi, \eta]
$$

for all vector fields $\xi$ and $\eta$. In the Christoffel symbol, this is equivalent to the symmetricity

$$
\Gamma_{i j}^{k}=\Gamma_{j i}^{k} \quad(\forall i, j, k)
$$

If a local section $\alpha=(u, v)=u d x+v d y$ of $\mathrm{T}^{*} \mathbf{R}^{2}$ is $\nabla^{*}$-parallel, then $u$ and $v$ satisfy

$$
\begin{aligned}
& 0=\left(\frac{\partial}{\partial x} u, \frac{\partial}{\partial x} v\right)+(u, v)\left(\begin{array}{ll}
\Gamma_{11}^{1} & \Gamma_{12}^{1} \\
\Gamma_{11}^{2} & \Gamma_{12}^{2}
\end{array}\right) \\
& 0=\left(\frac{\partial}{\partial y} u, \frac{\partial}{\partial y} v\right)+(u, v)\left(\begin{array}{ll}
\Gamma_{21}^{1} & \Gamma_{22}^{1} \\
\Gamma_{21}^{2} & \Gamma_{22}^{2}
\end{array}\right) .
\end{aligned}
$$


The symmetricity on $\left\{\Gamma_{i j}^{k}\right\}$ implies that

$$
\frac{\partial}{\partial y} u=\frac{\partial}{\partial x} v
$$

and thus the closedness of $\alpha$.

Suppose that $\nabla$ is flat and torsion free. Let $\alpha$ and $\beta$ be $\nabla^{*}$-parallel local sections of $\mathrm{T}^{*} \mathbf{R}^{2}$ on a simply connected nighborhood $U$ of the origin $(0,0)$. By Lemma 4.2, there exist functions $X$ and $Y$ on $U$ such that

$$
\alpha=d X, \quad \beta=d Y
$$

Define a map $\varphi: U \rightarrow \mathbf{R}^{2}$ by

$$
\varphi=(X, Y)
$$

If $d X$ and $d Y$ are linearly independent at one point on $U$, then they are so at all points.

Proposition 4.1. $\quad \nabla$-geodesics are transformed to lines by the above $\operatorname{map} \varphi$.

Proof. Since the transformation matrix $J(\varphi)$ is equal to $\left(\begin{array}{c}\alpha \\ \beta\end{array}\right)$, it follows, from the fact that $\alpha$ and $\beta$ are parallel, that $J(\varphi)$ satisfies

$$
d J(\varphi)+J(\varphi) \omega^{h}=0
$$

By multiplying $J(\varphi)^{-1}$ from the right, we obtain

$$
d J(\varphi) J(\varphi)^{-1}+\operatorname{Ad}(J(\varphi)) \omega^{h}=0 .
$$

This means that the horizontal component of the connection form that is obtained by the gauge transformation with respect to $J(\varphi)$ is equal to zero. Thus the corresponding Christoffel symbols all vanish. Therefore we conclude that all $\nabla$-geodesics are transformed into lines by the map $\varphi$.

Remark. Lemma 4.2 and Proposition 4.1 still hold for higher dimensional spaces. 


\subsection{Linearization of geodesics}

In the following, we will show that the graphs of all solutions of the equation

$$
y^{\prime \prime}+P+3 Q y^{\prime}+3 R\left(y^{\prime}\right)^{2}+S\left(y^{\prime}\right)^{3}=0
$$

are geodesics of a certain connection, and interpret the results in the previous sections using the connection.

Given smooth functions $P, Q, R$ and $S$ on $\mathbf{R}^{2}$, we associate a connection form $\omega$ whose horizontal component is equal to

$$
\begin{aligned}
\omega^{h} & =-\left(\begin{array}{cc}
\Gamma_{11}^{1} & \Gamma_{12}^{1} \\
\Gamma_{11}^{2} & \Gamma_{12}^{2}
\end{array}\right) d x-\left(\begin{array}{cc}
\Gamma_{21}^{1} & \Gamma_{22}^{1} \\
\Gamma_{21}^{2} & \Gamma_{22}^{2}
\end{array}\right) d y \\
& =\left(\begin{array}{cc}
Q & R \\
-P & -Q
\end{array}\right) d x+\left(\begin{array}{cc}
R & S \\
-Q & -R
\end{array}\right) d y
\end{aligned}
$$

and by $\nabla$ and $\nabla^{*}$ respectively the corresponding covariant differential and its dual. We remark that the connection form $\omega$ is $s l_{2}(\mathbf{R})$-valued and torsion free.

The following three lemma are verified by direct calculations:

Lemma 4.3. The graph of a function $y=y(x)$ is $\nabla$-geodesic, if and only if it satisfies the equation

$$
y^{\prime \prime}+P+3 Q y^{\prime}+3 R\left(y^{\prime}\right)^{2}+S\left(y^{\prime}\right)^{3}=0 .
$$

LEMma 4.4. $\omega$ is flat, if and only if $P, Q, R$ and $S$ satisfy

$$
\left\{\begin{array}{l}
P_{y}-Q_{x}=-2\left(P R-Q^{2}\right) \\
Q_{y}-R_{x}=-P S+Q R \\
R_{y}-S_{x}=-2\left(Q S-R^{2}\right) .
\end{array}\right.
$$

Lemma 4.5. For a function $X, d X$ is $\nabla^{*}$-parallel, if and only if $X$ satisfies

$$
\left\{\begin{array}{l}
X_{x x}=X_{y} P-X_{x} Q \\
X_{x y}=X_{y} Q-X_{x} R \\
X_{y y}=X_{y} R-X_{x} S .
\end{array}\right.
$$

Since $\omega$ is $s l_{2}(\mathbf{R})$-valued, the map $\varphi=(X, Y)$ constructed in Section 4.3 is area preserving. Now Theorem 2.1 is a corollary of Lemmas 4.3, 4.4 and 4.5 , and Propositions 4.1. 


\section{REFERENCES}

[1] M. A. Tresse, Détermination des invariants: ponctuels de de l'équation différentielle du second order $y^{\prime \prime}=w\left(x, y, y^{\prime}\right)$, Hirzel, Leipzig, 1896.

[2] É. Cartan, Sur les Variétés à Connecxion Projective, Bull. Soc. Math. France, 52 (1924), 205-241.

[3] Leçon sur la théorie des espaces à connexion projective, Gauthier-Villars, Paris, 1937.

[4] L La geometria de las ecuaciones diferencials de tercer orden, Rev. Mat. Hispano-Amer., t.4 (1941), 1-31 (Cuvres complètes, Partie III, Vol. 2, 1535-1565 Gauthier-Villars, Paris (1955)).

[5] N. Kamran, K. G. Lamb and W. F. Shadwick, The local equivalence problem for $d^{2} y / d x^{2}=F(x, y, d y / d x)$ and the Painlevé transcendents, J. Diff. Geom., 22 (1985), $139-150$.

[6] S. S. Chern, The geometry of a differential equation $Y^{\prime \prime \prime}=F\left(X, Y, Y^{\prime} Y^{\prime \prime}\right)$, Sci. Rep. Tsing Hua Univ., 1940, 97-111.

[7] H. Sato and A. Y. Yoshikawa, Third order ordinary differential equations and Legendre connections, J. Math. Soc. Japan, 50 (1998), No.4, 993-1013.

[8] - Projective contact structures on three-Dimensional manifolds, Preprint.

Tetsuya Ozawa

Department of Math. Meijo Univ.

Shiogamaguchi, Tenpaku-ku, Nagoya 468-8502

Japan

ozawa@meijo-u.ac.jp

Hajime Sato

Graduate School of Math. Nagoya Univ.

Chikusa-ku, Nagoya 464-8602

Japan

hsato@math.nagoya-u.ac.jp 